\title{
Research progress of EMT in Cancer Metastasis
}

\author{
Ruoyun $\mathrm{Du}^{1, *}$ \\ ${ }^{1}$ College of Chemistry and Materials Engineering, Beijing Technology and Business University, Beijing, 100048, China;
}

\begin{abstract}
Cancer is still a deadly disease that haunts people. Once there is a malignant tumor, patients need to receive a series of expensive, complex and painful treatments. Even so, cancer still causes high mortality, for its ability to easily metastasizing to other organs, without effective cures for any kind of cancer so far. Therefore, researchers have been trying to explore its mechanism and find ways to inhibit its development and discover new cure from various aspects. The latest research found that Epithelial-Mesenchymal Transitions (EMT) may be related to cancer, especially in the metastasis. It can be a breakthrough finding that render cancer a curable disease. In this paper, we systematically introduce the definition, classification, mechanism, influencing factors of EMT and its relationship with cancer metastasis and provide new angles and ideas for further exploring all levels of signal pathways and cancer treatment.
\end{abstract}

\section{Introduction}

Cancer is one of the most serious diseases because it can occur in any part of body, such as lung, breast, stomach. Even as, cancer survival rate, overall, is low[1]. Although there are many ways to treat cancer, most of them are not effective enough or the treatment process is very painful, and because the cancer is easy to spread and transfer to other parts of body, it is difficult to cure. Therefore, how to prevent and treat cancer has been a hot topic in the field of biology and medicine in recent years. The reason that cancer is more difficult to cure and can pose a greater threat to human is the infiltration of cancer cells, which enables cancer to metastasize and spread in the body very fast. This can lead to canceration of multiple organs, aggravate cancer, and reduce the possibility of recovery, which is why cancer mortality is so high. However, some researchers believe that the metastasis and spread of cancer cells may be related to Epithelial-Mesenchymal Transitions (EMT). EMT is a basic process of embryonic development and also the basis of wound healing. It involves cell morphological changes and cell migration, which is why researchers believe that EMT is closely related to cancer metastasis. Through the study of EMT, we can further explore the mechanism of cancer cell diffusion and metastasis and the influencing factors of this process, in order to find the means to control cancer metastasis.

\section{Overview of EMT}

\subsection{Definition of EMT}

\footnotetext{
*Corresponding author e-mail: 1701040103@st.btbu.edu.cn
}

Epithelial-Mesenchymal Transitions (EMT) is a controlled reversible process that has a shift between epithelial states and mesenchymal states. When epithelial cells transform into mesenchymal cells, it is called EMT; and when mesenchymal cells transform into epithelial cells, the process is called Mesenchymal-Epitheilial Transitions (MET). Epithelial cells have polarity and their state tends to be more stable, while the loss of polarity of mesenchymal cells makes their cellular state more transferable and diffusive. And inhibition of epithelial cell polarity and cell division can promote cell survival [2], which is also conducive to the transformation of epithelial cells into mesenchymal cells and transfer to other parts[3]. The important events that accompany the occurrence of EMT are cell morphological changes, destruction of basement membrane, de-epithelialization, loss of polarity and cell junction. Cancer metastasis is also related to those things, which makes EMT contribute to the spread of cancer cells.

\subsection{Classification of EMT}

There are three types of EMT. Primary EMT is related to implantation, embryo formation and organ development. Secondary EMT is related to wound healing, tissue regeneration, and organ fibrosis[4]. Tertiary EMT appears in vegetative tissue, which has undergone genetic and epigenetic changes, especially cell overgrowth and tumor development. These changes could mainly affect oncogenes and tumor suppressors[5]. Cancer cells become invasive and migrating after undergoing the third type of EMT, thus accelerating the progress of cancer[6]. After undergoing EMT, some cells still retain most of the characteristics of epithelial cells and only obtain some of the characteristics of mesenchymal cells, while some cells completely lose the characteristics of epithelial cells and 
become complete mesenchymal cells. The result of this transformation is that the cell structure is no longer neat and tight, but becomes loose, with fewer connections between cells, weaker adhesion and loss of polarity, all of which contribute to cell movement and transfer. Although this shift will occur and will lead to the above results, the exact mechanism is not clear[5].

\section{Mechanism of EMT}

Since EMT is a reversible process, when we study its mechanism, we must note that it can be regulated in many ways, and the most common of which are inducers and inhibitors. Inducers can promote the EMT process, while inhibitors can block it. As the occurrence of EMT has a positive impact on cancer metastasis, researchers have conducted in-depth studies on the inducers and inhibitors of EMT. It is expected that they can be used to regulate EMT and try to interfere with the metastasis and spread of cancer cells by affecting the process of EMT.

\subsection{Inducer}

Cadherin plays an important role in cell recognition, migration, tissue differentiation and adult tissue and organ composition in embryonic development. Among them, Ecadherin exists mainly in epidermis. The loss of Ecadherin expression will affect EMT or its reverse process and the development and metastasis of cancer to some extent[7]. This is because the down-regulation of Ecadherin reduces the intensity of cell adhesion in a tissue, resulting in increased cell activity. This allows cancer cells to penetrate the basement membrane and invade surrounding tissue. In the process of pathologist's diagnosis of cancer, the decrease or disappearance of Ecadherin expression is also one of the characteristics of tumor cell EMT. Therefore,E-cadherin can act as an inducer of the EMT process.

Inducers can act on the EMT process both intracellularly and extracellularly. In addition to the above-mentioned E-cadherin, some factors such as Pez, tyrosine phosphatase, PRL3 and IL-6 can induce EMT through different pathways[6]. Pez is different from other molecules in that it can be induced by TGF- $\beta$ and then induce MDCK cells to regulate EMT by triggering Snail and Zeb, and it can also induce the production of TGF- $\beta$ to promote the occurrence of an autocrine circuit[6]. Other factors have different degrees of inducing effects on different cancer cell lines. Tyrosine phosphatase induces EMT of colon cancer cells by activating PI3K/AKJ[6]. PRL3 induces EMT by activating Src in the kidney cell line[8]. The cytoplasmic kinase, Aurora-A induces EMT in nasopharyngeal tumor cells through the MAPK pathway. Snail induces the expression of IL-6, and promotes the EMT process of breast cancer cells through IL-6[6].

\subsection{Inhibitor}

MicroRNAs, growth factors and signaling pathways can suppress EMT by inhibiting the transcription of EMT transcription factors (Snail, Slug, Twist, ZEB1)[9].Transcription factors can change the activity of genes that play a role in the cell division cycle, which may be an important factor in carcinogenesis. The activation or inhibition of many transcription factors is controlled by signal transduction pathways. Cofactors also regulate them by forming complexes that activate or inhibit transcriptional activity. For example, Notch and Wnt can also affect the expression of EMT transcription factors. The transcription factor of MET can inhibit the expression of E-cadherin. When its expression is reduced, it will reduce the intensity of cell adhesion in the tissue, resulting in increased cell activity, allowing cancer cells to better metastasize, spread and infiltrate.

\subsection{EMT Signaling Pathways}

A large number of signal transduction pathways and substances can induce or inhibit the EMT process in cells. Therefore, we summarize the findings on how snails and E-cadherin are affected by factors that regulate the EMT process. Through these work, we can realize that regulating the signal pathway of EMT can indeed have an impact on the metastasis and spread of cancer and Table 1 shows the influence factors and signal pathways that can promote or inhibit the EMT process.

Table 1. EMT Signaling Pathways.

\begin{tabular}{cc}
\hline Signaling pathways and agents & Impact on EMT \\
\hline FGF & promote \\
HGF & promote \\
PDGF & promote \\
IGF & promote \\
PGE(2)/COX2 & promote \\
VEGF & promote \\
Estrogen & inhibition \\
Hypoxia/AMF & promote \\
Notch & promote \\
PTH(rP)R & promote \\
Bile acids & promote \\
Nicotine, UV & promote \\
Integrins & promote \\
Wnt & promote \\
TGF- $\beta$ & promote \\
\hline
\end{tabular}




\begin{tabular}{cc}
\hline ILEI, IL-6 & promote \\
Shh & promote \\
BMP & promote \\
SCF & promote \\
EGF & promote \\
PAK-1, LOXL, SCP, TNF- $\alpha$, YB-1 & promote \\
Gsk-3-mediated phosphorylation & inhibition \\
\hline
\end{tabular}

Notes. Retrieved from Ref. [1].

First, NF-kB promotes the stability of Snaill protein and prevents it from being phosphorylated and degraded by GSK-3[10]. Conversely, if wild-type p53, ubiquitin ligase MDM2 and Snail2 form a ternary complex, it will trigger degradation[11]. There are two proteins associated with breast cancer invasion, both of which are related to Snail. The first protein is Y-box binding protein (YB-1), which acts as a translation regulator to activate Snail mRNA translation[12]. Another protein zinc transporter LIV1 can increase Snail nuclear translocation, thereby inducing EMT and promoting the aggressiveness of tumor cells[12]. Snail, as a multidirectional protein, may require tissue-specific binders to coordinate to regulate transcription, such as Ajuba, LIMD 1 and WTIP, which are collectively called Ajuba Lim proteins[13]. In addition to these, it can also bind to Smad protein. Smad protein plays a core inhibitory effect in EMT caused by TGF- $\beta$, and its inhibitory effect disappears after being bound by Snail, which is beneficial to cell migration[14]. Snail plays a very important role not only in breast cancer, but also in other cancers. For example, in colon cancer, Snail 1 and Zeb 1 also play a vital role. Whether it can function normally is regulated by the expression levels of transcription core regulators GBP and p300[15]. Snail can also increase the Wnt signal by reducing the content of $\mathrm{E}$ cadherin and increasing the $\beta$-catenin in the cytoplasm and the connection disorder, or it can also directly promote the Wnt signal enhancement by interacting with $\beta$-catenin[16]. Since people have discovered that Snail 1 can activate Zeb 1 , the activation process of Snail is always accompanied by the activation of the Zeb gene, but studies have found that Zeb is still active in some tumors that do not express Snail[15], besides, the cell environment is different. Under the circumstances, different inducers will be provided. Therefore, we should analyze the regulation of Zeb expression independently.

E-cadherin inhibitor as an EMT inducer can regulate the expression of a variety of genes, which inhibits epithelial traits and promotes the interstitial state. It can promote cell survival by inhibiting the polarity and cell division of epithelial cells. Attenuation of cell proliferation is conducive to invasion and tumor growth, while resistance to cell death confers the advantage of embryo migration or tumor invasion cells to distant organs. The above-mentioned mechanism does not strictly inhibit the expression of E-cadherin but is a regulator of the adhesion movement of epithelial phenotype cells. EMT inducers that indirectly inhibit the transcription of Ecadherin often activate some direct inhibitors with several targets to influence regulation in multiple pathways. Kruppel-like factor 8 (KFL8) directly binds to the Ecadherin promoter through GT boxes to promote EMT and cancer cell invasion into breast cell lines and lymph[17]. Twist and Goosecoid can indirectly reduce E-cadherin. FoxC2 is downstream of Twist, Snail and Goosecoid, they do not affect the expression of cadherin and can promote cytoplasmic localization[18]. Its main role may be the induction of mesenchymal properties and Twist, FoxC2 and Goosecoid are all related to metastatic potential[18].

In addition to the two core factors mentioned above, the normal operation of EMT signaling pathway is inseparable from the execution of EMT-activated transcription factor (EMT-TF). EMT-TF can stimulate cell movement and keep cells dry and increase tumorigenicity, indicating that EMT plays a role in tumor formation and precursor lesion formation[19]. In addition, EMT-TF is also involved in double-stranded DNA repair, which allows cancer cells to escape senescence and also induces the expression of anti-apoptotic genes[19]. In Tertiary EMT, EMT-TF mainly includes Snail, Twist, and Zeb and they plays an important role at all stages from tumor growth, invasion and metastasis to colonization, as well as treatment resistance.

\section{Causes of EMT-related cancer cell evolution}

The relationship between EMT and the evolution of cancer cells is reflected in many aspects: First, EMT can give cells a high degree of anti-apoptosis ability and can induce non-cancer stem cells to enter a cancer stem celllike state[19]. Such cells have non-senescence, continuous division and differentiation. It has the potential to form tumors, especially after the cancer metastasizes, it can become a source of new cancers[19]. Second, the expression of EMT-related genes can be detected at the edge of the local invasion of the primary tumor. In addition, EMT generated by tumor cells can also increase their ability to pass through vascular endothelial cells into the blood circulation, which indicates that the occurrence of EMT is closely related to the early stage of tumor metastasis[19]. In tumor metastasis, the down-regulation of the cell junction complex is often accompanied by the EMT process. These enhance the credibility of EMT's view that it is related to the metastasis and spread of cancer.

In the past two decades, the application and exploration of EMT in the field of cancer has aroused people's interest in order to study the pathological process of tumor metastasis and find a new perspective for the treatment of cancer. Although researchers have different views on whether EMT plays a role in cancer metastasis, more data have been found that we need to explore more deeply the role of EMT in cancer progression. Therefore, 
it is necessary to discuss the latest development of EMT biological processes.

\subsection{EMT and Cancer Stem Cells}

EMT is activated in cancer cells to facilitate its separation from the primary tumor and its penetration into the blood to spread to other parts of the body. Each type of cancer has a different tendency to EMT, thus showing different EMT states[6]. By studying the state of EMT or the state of cancer stem cells, we can correspondingly try to control the spread and metastasis of cancer cells in order to curb cancer, and it has played a positive role in the treatment of cancer, and provides a bright prospect for curing cancer. EMT is closely related to the spread of invasive tumors and the characteristics of stem cells, such as the ability of self-renewal, infinite division, proliferation and multidirectional differentiation potential, and can differentiate into different types of tissue cells; that is, it has the plasticity of differentiation and development, and can be induced to differentiate into cell types that are not related to development in a specific environment. When tumor stem cells have these characteristics and the EMT process is activated in cancer cells, both of them have a positive effect on the metastasis and spread of cancer. There are many genes or pathways that regulate EMT, Notch and NF. Both $\kappa$ B and HIF1/2 affect EMT through Snail1/24[6]. They have a synergistic effect on the metastasis of tumor stem cells by affecting EMT.

\subsection{Metastatic cascade}

EMT is thought to contribute to the metastatic cascade by making invasive cancer epithelial cells lose polarity and detach from the basement membrane, and then promote tumor malignant growth by regulating angiogenic switches, eventually enabling cancer cells to enter circulation and withdraw from the bloodstream at other distant locations[20]. Through some transferases, they can reverse from mesenchymal to epithelial phenotype, thus completing the metastasis and spread of cancer cells. However, certain stromal cells, such as cancer-associated fibroblasts(CAFS) [21], immune cells [22], tumorassociated macrophages [23] and other bone marrowderived cells[24], must be collected before the cancer cells from the primary tumor can be transferred to other parts of the blood. After preparing for the transfer, the plasticity of the cells derived from the primary tumor makes the metastatic tumor cells epithelioid. Mesenchymal cells need to return to epithelial phenotype when they want to settle in a new destination[25]. Before that, mesenchymallike cells had better adaptability, reflected in better drug resistance[26], resistance to shear stress and adaptation to cell metamorphosis[27], which helped cancer cells to carry out their metastasis cascade process to facilitate metastasis and spread in the body[25].

However, whether EMT is the basic element of transfer cascade still needs to be discussed. Although many cell experiments and animal experiments provide evidence of the existence of EMT in primary tumor cells, they do not contribute significantly to the formation of cancer cell metastasis and growth[28]. But according to the relevant experimental results released by other researchers, we cannot rule out EMT as a driver of cancer progression[29].

\section{Challenges, Future Directions and Perspectives}

The difficulty now is to explore whether EMT is directly related to the metastasis and spread of cancer, determining the role of EMT in the metastasis cascade and what is the mechanism of its physiological and biochemical processes. The answer to those questions is urgent for all parties to find more evidence to support their views. In addition, epigenetics has been very popular in scientific research in recent years, so I think the focus of future research is to study in more detail the regulatory factors that regulate the intermediate state between EMT and epigenetics and the role of EMT in the transfer cascade[30]. Now some scholars have found that epigenetic modifying enzymes can coordinate with EMT-related transcription factors. Through the in-depth study of the above directions, we can have a more comprehensive understanding of EMT and try to find new ideas and treatments for the prevention and treatment of cancer metastasis.

\section{References}

1. Siegel, R.L., K.D. Miller, and A. Jemal, Cancer statistics, 2020. CA: A Cancer Journal for Clinicians, 2020. 70(1): p. 7-30.

2. Peinado, H., D. Olmeda, and A. Cano, Snail, ZEB and bHLH factors in tumour progression: an alliance against the epithelial phenotype? Nature Reviews Cancer, 2007. 7(6): p. 415-428.

3. Barrallo-Gimeno, A. and M.A. Nieto, The Snail genes as inducers of cell movement and survival: implications in development and cancer. Development, 2005. 132(14): p. 3151-3161.

4. Zeisberg, M. and E.G. Neilson, Biomarkers for epithelial-mesenchymal transitions. J Clin Invest, 2009. 119(6): p. 1429-37.

5. Zhang, X.-H. and Z.-M. Yang, Epithelialmesenchymal Transition During Tumor Metastasis, Embryonic Development and Female Mammalian Reproduction. Progress in Biochemistry and Biophysics, 2012. 39(04): p. 307-313.

6. Thiery, J.P., et al., Epithelial-mesenchymal transitions in development and disease. Cell, 2009. 139(5): p. 871-90.

7. Singh, A. and J. Settleman, EMT, cancer stem cells and drug resistance: an emerging axis of evil in the war on cancer. Oncogene, 2010. 29(34): p. 47414751 .

8. Liang, F.B., et al., PRL3 promotes cell invasion and proliferation by down-regulation of Csk 
leading to Src activation. Journal of Biological Chemistry, 2007. 282(8): p. 5413-5419.

9. Lee, J.M., et al., The epithelial-mesenchymal transition: new insights in signaling, development, and disease. J Cell Biol, 2006. 172(7): p. 973-81.

10. Wu, Y., B.M. Evers, and B.P. Zhou, Small Cterminal Domain Phosphatase Enhances Snail Activity through Dephosphorylation. Journal of Biological Chemistry, 2009. 284(1): p. 640-648.

11. Wang, S.-P., et al., p53 controls cancer cell invasion by inducing the MDM2-mediated degradation of Slug. Nature Cell Biology, 2009. 11(6): p. 694-704.

12. Evdokimova, V., et al., Translational Activation of Snail1 and Other Developmentally Regulated Transcription Factors by YB-1 Promotes an Epithelial-Mesenchymal Transition. Cancer Cell, 2009. 15(5): p. 402-415.

13. Langer, E.M., et al., Ajuba LIM proteins are Snail/Slug corepressors required for neural crest development in Xenopus. Developmental Cell, 2008. 14(3): p. 424-436.

14. Vincent, T., et al., A SNAIL1-SMAD3/4 transcriptional repressor complex promotes TGFbeta mediated epithelial-mesenchymal transition. Nature Cell Biology, 2009. 11(8): p. 943-U84.

15. Pena, C., et al., The expression levels of the transcriptional regulators $\mathrm{p} 300$ and $\mathrm{CtBP}$ modulate the correlations between SNAIL, ZEB1, Ecadherin and vitamin $\mathrm{D}$ receptor in human colon carcinomas. International Journal of Cancer, 2006. 119(9): p. 2098-2104.

16. Stemmer, V., et al., Snail promotes Wnt target gene expression and interacts with beta-catenin. Oncogene, 2008. 27(37): p. 5075-5080.

17. Wang, X., et al., Kruppel-like factor 8 induces epithelial to mesenchymal transition and epithelial cell invasion. Cancer Research, 2007. 67(15): p. 7184-7193.

18. Yang, J. and R.A. Weinberg, Epithelialmesenchymal transition: At the crossroads of development and tumor metastasis. Developmental Cell, 2008. 14(6): p. 818-829.

19. Brabletz, T., et al., EMT in cancer. Nature Reviews Cancer, 2018. 18(2): p. 128-134.

20. Kalluri, R. and R.A. Weinberg, The basics of epithelial-mesenchymal transition. J Clin Invest, 2009. 119(6): p. 1420-8.

21. Lau, Eunice Yuen T., et al., Cancer-Associated Fibroblasts Regulate Tumor-Initiating Cell Plasticity in Hepatocellular Carcinoma through cMet/FRA1/HEY1 Signaling. Cell Reports, 2016. 15(6): p. 1175-1189.

22. Gentles, A.J., et al., The prognostic landscape of genes and infiltrating immune cells across human cancers. Nature Medicine, 2015. 21(8): p. 938-945.
23. Hsu, Dennis S.-S., et al., Acetylation of Snail Modulates the Cytokinome of Cancer Cells to Enhance the Recruitment of Macrophages. Cancer Cell, 2014. 26(4): p. 534-548.

24. Barcellos-Hoff, M.H., D. Lyden, and T.C. Wang, The evolution of the cancer niche during multistage carcinogenesis. Nature Reviews Cancer, 2013. 13(7): p. 511-518.

25. Giampieri, S., et al., Localized and reversible TGF $\beta$ signalling switches breast cancer cells from cohesive to single cell motility. Nature Cell Biology, 2009. 11(11): p. 1287-1296.

26. Aceto, N., et al., Circulating Tumor Cell Clusters Are Oligoclonal Precursors of Breast Cancer Metastasis. Cell, 2014. 158(5): p. 1110-1122.

27. Au, S.H., et al., Clusters of circulating tumor cells traverse capillary-sized vessels. Proceedings of the National Academy of Sciences, 2016. 113(18): p. 4947-4952.

28. Zheng, X., et al., Epithelial-to-mesenchymal transition is dispensable for metastasis but induces chemoresistance in pancreatic cancer. Nature, 2015. 527(7579): p. 525-530.

29. Maheswaran, S. and D.A. Haber, Transition loses its invasive edge. Nature, 2015. 527(7579): p. 452453.

30. Zhang, J. and H. Li, The Epigenetic Regulation of Epithelial-Mesenchymal Transition in Cancer Progression. Integrated technology, 2015. 4(04): p. 55-63. 